\title{
UTILIZATION OF METADATA FOR QUALITY ASSURANCE IN THE INTERNATIONAL SHARING AND REUSE OF OPEN EDUCATIONAL RESOURCES
}

\author{
Tsuneo Yamada (yamada@code.u-air.ac.jp) \\ Yosuke Morimoto (morimoto@code.u-air.ac.jp) \\ Center of ICT and Distance Education (CODE), \\ Open University of Japan (OUJ), Japan
}

\begin{abstract}
S
In order to sustain the development and utilization of learning content under the limited financial and human resources, the sharing and reuse of open educational resources (OERs) have been promoted in various school levels and disciplines. In many countries, central organization(s) collect both learning content and its metadata, and provide them to learners and teachers with distinctive value-added services from their portals. Collaborations among the national portals have also emerged beyond borders. Global Learning Object Brokered Exchange (GLOBE) is an international consortium of the hub organizations, which manage a federated repository and/or a meta-referatory in each country and region. GLOBE was established in September 2004 by five founding members, and now twelve organizations in the world participate in. By adopting IEEE LOM version 1.0 for the metadata standards, SQI for the query language, and OAI-PMH for harvesting, GLOBE realized a global search and delivery infrastructure for lifelong learning (LLL). At present, while the repositories all over the world store huge number of learning content and metadata, the variance of the quality becomes much bigger. In addition, because the effectiveness of learning content is relative and dependent on contextual factors, both optimizations specialized in learner characteristics and localizations to each language/culture are indispensable. The users need some supports on the quality and pedagogical guidance when they find their right content. GLOBE, based on multilingualism, multiculturalism and pluralism, collaborates to build up standardized services on the quality assurance of the content utilizing the "Educational" items of LOM. In AAOU framework also, the organizations can promote "openness", and share the quality learning content and educational information by using a standardized content search and delivery infrastructure.
\end{abstract}

Keywords: MERLOT, OER metadata, quality assurance, TELUQ.

With the innovation of information and communication technologies (ICT) and the spread of digital media environments, the educational uses of ICT are promoted in various stages of Life Long Learning (LLL). One of the critical factors is the accumulation and utilization of high-quality digital learning content. In order to sustain the development under the limitations of human and financial resources, some strategies on the sharing and reuse of learning content are indispensable. While the "open educational resources (OER)" movement is one of such development/distribution models (OECD, 2007; D'Antoni, 2007), "learning object" is a technical concept of learning materials to realize it. 


\section{OER MOVEMENTS}

While "openness" has been one of the essential questions in the education (e.g. liyoshi \& Kumar, 2008), the new deployments were proposed in the midst of ICT revolutions in this century. Harmonizing sometimes with "open source" and "open standards" initiatives, "open content" has been collected, shared and opened to the public in various OER movements. Open Courseware (OCWC, http: //ocwconsortium.org/) is the leading OER initiative, which was originally advocated by Massachusetts Institute of Technology (MIT). Currently, more than 200 higher education institutions and associated organizations participate in the consortium from more than 25 countries. As, in their original concept, $\mathrm{OCW}$ is not the sharing of MIT's education itself but that of course-related materials, many OER initiatives followed it proposing the new concepts and features; "Connexions" (launched by Rice University in 1999, http://cnx.org/) and "Open Learning Initiatives" (OLI, launched by Carnegie Mellon University in 2002, http://oli.web.cmu.edu/openlearning/) are the examples focusing more on the reusability, quality assurance and pedagogical considerations.

In the realm of open education, all the content of open universities has not been open to the public because of the sustainability. Now, in the trends of OER, the Open University in the UK launched "Open Learn" and Open University the Netherlands (OUNL) started a new framework for the mission.

\section{GLOBAL INFRASTRUCTURE FOR SHARING AND REUSE OF QUALITY LEARNING CONTENT}

In the last ten years, tremendous number of learning content was accumulated in uncountable repositories all over the world. They have huge diversity in quality, right management, pedagogical and instructional features, linguistic and cultural backgrounds and so on. As our financial and human resources are limited for the development and the maintenance, we should find the right content from such collections and reuse it before we develop the new by ourselves.

As of 2004, the collaborations among domestic institutions which managed learning content repositories and/or metadata referatories were realized successfully in several countries or regions. For example, edu Source Canada project was to construct a Pan-Canadian umbrella organization for linking provincial repositories / referatories.

In order to facilitate the international exchange and sharing of quality learning content, the national hub organization in each country and region, which manages the functions for federated repositories and meta-referatory, established "Global Learning Objects Brokered Exchange (GLOBE)" consortium in the September of 2004.

The original members were ARIADNE (Alliance of Remote Instructional Authoring \& Distribution Networks for Europe, http://www.ariadne-eu.org/, EU), education.au limited (http://www.edna.edu.au/, Australia), eduSource Canada (McGreal, R. et. al, 2004), MERLOT (the Multimedia Educational Resource for Learning and Online Teaching, http://www.merlot.org/, North America), and National Institute of Multimedia Education (NIME, Japan). NIME constructed a nationwide infrastructure for sharing and distributing digital learning content, called "NIME-glad (Gateway to Learning for Ability Development)". Now, the number of GLOBE members is twelve; eduSourceCanada was replaced with 
LORNET (http://www.lornet.org/) in February 2006 and NIME was merged into the Open University of Japan as Center of ICT and Distance Education (OUJ-CODE, http://www.code.u-air.ac.jp/) in April 2009. Korea Educational Research and Information Services (KERIS, http://english.keris.or.kr/, Korea), European Schoolnet (http://www.europeanschoolnet.org/, EU), the Center for Open Sustainable Learning, Utah State University (COSL, http://cosl.usu.edu/, USA), Latin-American Community of Learning Objects (LACLO, http://www.laclo.espol.edu.ec/, Latin America) joined GLOBE in 2007, Institute for Information Industry (III, http://www.iii.org.tw/english/, Taiwan) and Institute for the Study of Knowledge Management in Education (ISKME, http://www.iskme.org/, USA) in 2008, and Thailand Cyber University Project (TCU, www.thaicyberu.go.th, Thailand) in 2009. By adopting IEEE-LOM (Learning Object Metadata) version 1.0 (IEEE, 2002) for the metadata standards, Simple Query Interface (SQl; Simon, Massart, Van Assche, Ternier \& Duval, 2005) for the query language, and Open Archive Initiative Protocol for Metadata Harvesting (Logoze, Van de Sompel, Nelson \& Warner, 2002) for harvesting, GLOBE realized a global search and delivery network. GLOBE also harmonizes with "Open Standards" concept and some of the members join international standardization activities, such as, Learning Object Discovery and Exchange (LODE) Project at IMS-Global Learning Consortium (IMS/GLC, http://www.imsglobal.org/).

\section{AN IMPLEMENTATION OF GLOBE GLOBAL SEARCH IN JAPAN}

Learning content is stored and managed in institutional or personal repositories on the Internet. Without some cross-institutional search mechanism, it is difficult for teachers and learners to find and retrieve the appropriate content from the deep Web on the globe.

\section{AGGREGATION OF METADATA AT A NATIONAL PORTAL FOR JAPANESE HE RESOURCES}

When NIME planned and constructed initially "NIME-glad" portal, most of Japanese HE institutions had neither institutional repositories nor content management systems in which they could store their own educational/learning content. In addition, many institutions and faculties were reluctant to manage them with metadata by themselves. NIME tagged metadata to their learning content and accumulated them at NIME referatory in a simple "aggregation" way (Figure 1). The goal of the system is, by accumulating metadata at a database, to search learning content cross-institutionally.

\section{FEDERATED SEARCH NETWORK WITH GLOBE MEMBERS}

Federated search is one of the cross-database search methodologies without sharing the full set of or a part of the metadata among database(s). When GLOBE was established in 2004, the member organizations could not share the full metadata set with others because they were not always the copyright holder of the metadata. GLOBE adopted a federated search architecture and connected in "one-to-one correspondence" fashion using Simple Query Interface (SQI) as the query language.

When a search system ("NIME-glad" search system in Figure 2) receives the keyword(s) from a user (a), the federated search client ("FS Client") of the system sends the queries both to its own metadata database (that is, the referatory; b) and to each federated search server ("FS Server") of other organizations (c). Each remote search system searches using the keywords sent, and returns the search results (d). The original search system merges all of the results in a local rule and sends back to the user's terminal (e). As a result, the user can get the cross-institutional search results in an integrated manner. At this stage, the most of 
the registered learning content was OER.

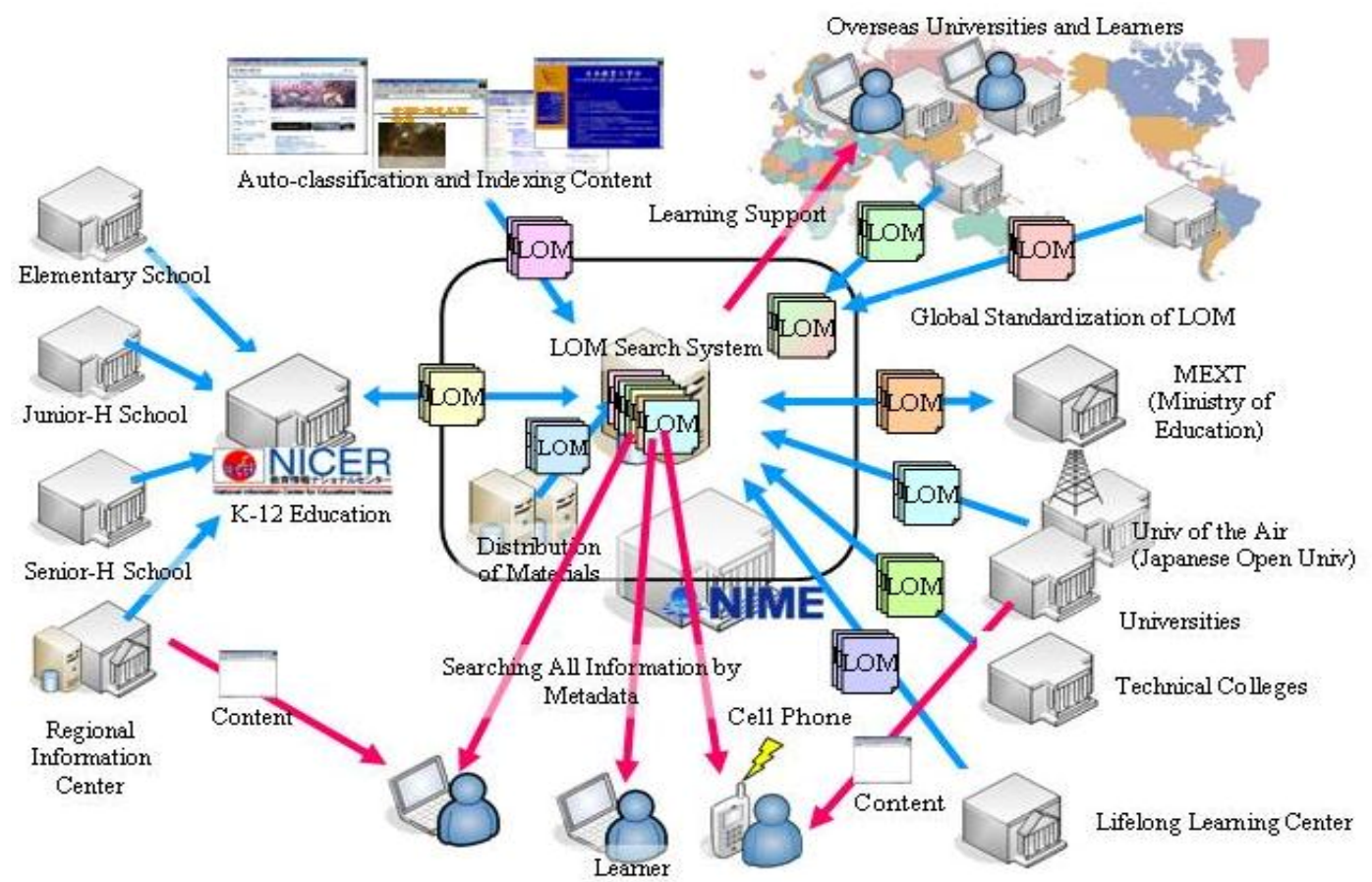

Figure 1. Grand design of "NIME-glad (Gateway to Learning for Ability Development)" (cf. NIME handbook, 2004-2008)

A Japanese user's terminal (a) Send keywords

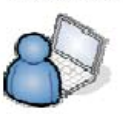

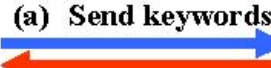

(e) Merge and display the search results

(b) Send a query

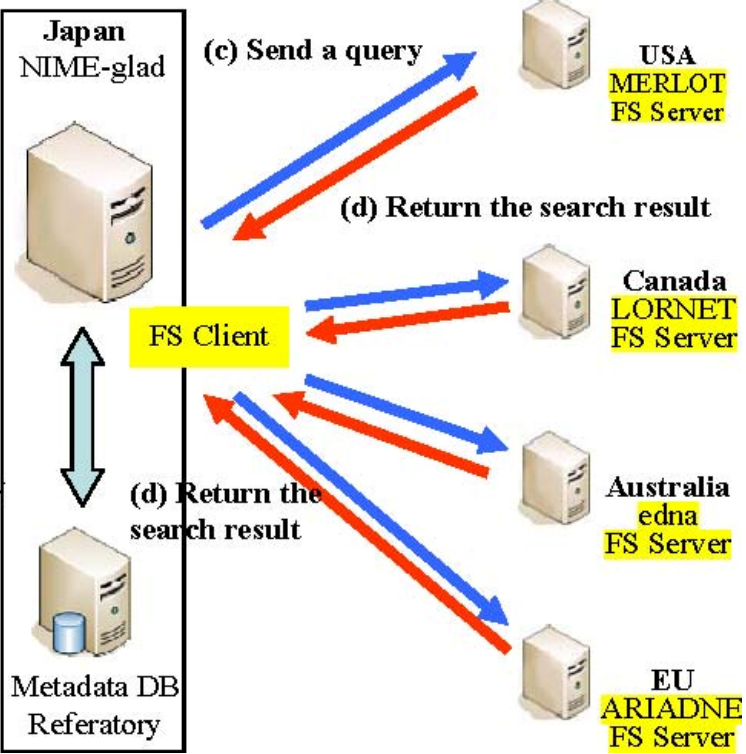

Figure 2. A data flow at GLOBE global federated search (Yoshii, Shimizu \& Yamada, 2008)

\section{HARVESTING AND ADVANCED ARCHITECTURE FOR THE NEXT-GENERATION GLOBE SEARCH}

When the number of GLOBE member organizations is increasing, the "one-to-one correspondence" strategy induces serious problems. When a member changes a part of the 
specification, other members have to cope with the changes each time. When every new member joins, each original member must develop the new specific interface. Now, GLOBE needs some more scalable and interoperable architecture for the next advanced search. In February 2007, harvesting (cf. OAI-PMH) was added as a supplementary prerequisite for the participation. After the closing of NIME, OUJ-CODE has also begun to implement a harvesting system for the GLOBE search functions. In addition, several GLOBE members, that is, European Schoolnet, LORNET and NIME join LODE project at IMS/GLC and prepares for the new international standards on federation of repositories.

The main function of GLOBE search at the first stage was to show the location of the searched content. Now, moving to the second stage, the portals and gateways are requested to have some distinctive value-added services, that is, to provide additional information and services on rights management, quality assurance, education/pedagogical supports or recommendation, localization and so on. For example, California State University System, which is the founder of MERLOT, one of GLOBE member, launched "Digital Marketplace (DM, http://www.calstate.edu/ats/digital_marketplace/)" Initiative, which covers both proprietary content and open content. At the moment, several GLOBE members can accept the exchange the rights information. From the backgrounds, GLOBE has multilingual, multicultural and pluralistic orientations. GLOBE needs a more inclusive and interoperable architecture, based on the demand analysis of present/potential users and on the latest trends in international standardization activities.

\section{QUALITY ASSURANCE ISSUES AND THE SOLUTIONS}

The quality of learning content and its metadata is one of the critical factors for the sharing and distribution, and it is considered that the information on the quality will be one of the basic and pressing value-added services of the global portals and international gateways. MERLOT operates an excellent peer-review and recommendation system for the HE learning content. TELUQ (Télé-université, the Distance University of Quebec) launched "the Quality for Reuse (Q4R)" project under the collaborations with Canadian universities and GLOBE. Q4R project "aims at fulfilling this vision by providing tools, techniques, procedures, principles and strategies assisting in implementing quality assurance practices for high quality LOR (learning object repositories; http://www.q4r.org/)". In general, the organizations focusing on K-12 education are more sensitive in the quality issues while others have often only minimum standards (OECD, 2001). In many cross-institutional search services, both selection of query results and display order depend on the local rule of each member. In this process, high-quality metadata which include the information of the content quality will be preferable.

\section{EXTENSION OF METADATA ITEMS FOR QUALITY ASSURANCE IN JAPAN}

After the merger into OUJ in April 2009, we are reorganizing the portal and the global search services in the new framework of open education and lifelong learning (LLL). We are renaming and redesigning them for new value-added services. In realizing new advanced search functions, which are optimized to LL learners' characteristics and met with their new demands, it is necessary to utilize more metadata elements and to describe various attributes in a standardized fashion. By utilizing new elements, we can start new innovative value-added services and improve the quality of the services.

While the number of LOM items used in NIME-glad was thirty-seven, the number of items 
which were exchanged in GLOBE federated search network was limited to four, that is, location (URLs), title, abstracts and key words. We extend several metadata items to describe more information on rights, quality, educational/pedagogical recommendation and localization. As some GLOBE members have already implemented their own quality standards, the description on metadata should be also relative and contains both a referred guideline(s) and the results of the evaluation. GLOBE can collaborate to build up standardized services on the quality assurance of the content utilizing the "Educational" items of LOM.

In Japan, Accreditation Council for Practical Abilities (ACPA, http://www.acpa.jp/en/) has started unique evaluation activities. ACPA is a non-profit organization established in 2003 with the support of the government, corporations and HE institutions. In order to assure the quality of practical learning resources and to promote the exchange especially between universities and corporations, ACPA defines the practical abilities, develops the standard skill matrix in each field, and performs certification and accreditation of courses, course providers, educational institutions, lecturers, and trainees based on their original or standardized criteria. The standard skill matrix on ICT domain and Business and Management domain are open to the public (ICT domain:

http://www.acpa.jp/en/acpa/standard_skill_matrix_bmd.pdf). We are redesigning our metadata structure in order to describe both the evaluation source and its results. For example, the category field of "Quality" item shows the name of evaluation organization and its standards, and the value field shows the result of the evaluation. Under the collaboration with ACPA, we are revising the set of our metadata (cf. Item 20 "Quality: Category-Value", Table 1).

Table 1. LOM items in the new OUJ-CODE search system (partially under development)

\begin{tabular}{|c|c|c|c|c|}
\hline \multicolumn{2}{|c|}{ OUJ-CODE metadata } & \multicolumn{2}{|l|}{ IEEE LOM1.0 } & \multirow{2}{*}{$\begin{array}{l}\text { Differentials } \\
\text { from NIME }\end{array}$} \\
\hline 1 & ID of metadata & & & \\
\hline 2 & ID of Learning Object & 1.General & 1.1 Identifier & \\
\hline 3 & Title & & 1.2 Title & \\
\hline 4 & Language of LO & & 1.3 Language & \\
\hline 5 & Description & & 1.4 Description & \\
\hline 6 & Keywords & & 1.5 Keyword & \\
\hline 7 & Aggregation Level & & 1.8 Aggregation level & \\
\hline 8 & Contributors & 2.Life cycle & 2.3 Contributor & \\
\hline 9 & Language of metadata & 3.Meta metadata & 3.4 Language & \\
\hline 10 & Technical-Format & 4.Technical & 4.1 Format & \\
\hline 11 & URL & & 4.3 Location & \\
\hline 12 & Technical-Requirements & & 4.4 Requirement & \\
\hline 13 & Educational-Context & 5.Educational & 5.6 Context & \\
\hline 14 & Educational-Typical Learning Time & & 5.9 Typical learning time & \\
\hline 15 & Educational-Description & & 5.10 Description & \\
\hline 16 & Rights- Cost & 6.Rights & 6.1 Cost & \\
\hline 17 & Rights-Description & & 6.3 Description & \\
\hline 18 & Classification & 9.Classification & - & \\
\hline 19 & Rights: Category-Value & Not fixed & & NEW \\
\hline 20 & Quality: Category-Value & Not fixed & & NEW \\
\hline 21 & Access Control & Not fixed & & NEW \\
\hline 22 & Harvesting & Not fixed & & For GLOBE \\
\hline 23 & Federated Search & Not fixed & & $\bar{B}$ \\
\hline
\end{tabular}




\section{CONCLUSION}

In order to sustain the development under the limitations of human and financial resources, some strategies on the sharing and reuse of learning content are indispensable. The teachers, content developers, course providers and policy-makers can compare and analyze the quality of the existing content and, by reusing them, may save their own limited resources. Lifelong learners, who are diversified in various attributes, may find more appropriate learning content for their continuous education from the world.

Considering international collaborations on the development and distribution of quality learning resources, some global cross-institutional search system will be effective. In order to promote such global exchange and delivery among repositories, international standards are necessary in various aspects, such as metadata, query language and other specifications for federations of repositories.

\section{Internationalization and localization}

When we utilize the learning content cross the borders, we must consider localization processes to adapt them to new local learners' characteristics and learning environments. In our international collaborative researches (in preparation), the differences in various aspects, that is, in languages, social customs, school systems, curricula and standards, pedagogies, ICT environments, learner characteristics and so on, were found, and it was suggested that we should optimize the content in order to avoid unnecessary cultural frictions. Educational institutions and their teachers' communities have such the knowledge and solutions for the localization, and can contribute to the learners' communities by adding their recommendation to the metadata.

\section{Collaborations among open universities}

While the learning content at open universities is not always open to the public, some OUs provide their learning content as OERs. The Open University UK launched "Open Learn" project (http://openlearn.open.ac.uk/) and European Association of Distance Teaching Universities (EADTU) coordinates "Multilingual Open Resources for Independent Learning (MORIL, http://moril.eadtu.nl/) gateway.

The open universities in Asia and other regions develop huge number of courseware and its materials every year and have stored them at their repositories. Although they cannot open all of their learning content because of the sustainability, if they co-develop some federation system of the repositories, their valuable digital resources become visible and the utilization will be promoted among open universities. Furthermore, by connecting it with some international brokerage system for discovery and delivery, such as GLOBE, OUs' content will be sharable with non-OU lifelong learning communities and OUs can contribute more to the global knowledge-based society. In addition, OUs, as regional centers of lifelong learning (LLL), accumulate the knowledge for the personalization and localization of learning, and can support autonomous learning of $\mathrm{LL}$ learners by combining their recommendation with the metadata search system.

"Openness" is one of the main trends in both open and traditional universities. In AAOU framework, the organizations can promote the concept and share the "quality" learning content and educational information using standardized search and exchange frameworks. 


\section{ACKNOWLEDGMENTS}

This study is partially supported by Grant-in-Aid for Scientific Research (A) to the authors (Grant No. 20240072). This study is based on collaborative researches and/or operations with GLOBE partner organizations and Accreditation Council for Practical Abilities (ACPA) in Japan.

\section{REFERENCES}

D'Antoni, S. (2007). Sharing content: access to knowledge, International Institute for Educational Planning Newsletter, Vol. XXV, and $\mathrm{N}^{\circ} 2$, http://www.unesco.org/iiep/eng/newsletter/2007/apre07.pdf.

IEEE (2002). Draft standard for learning object metadata (IEEE 1484.12.1-2002).

liyoshi, T., \& Kumar, V. (2008). Opening up education: The collective advancement of education through open technology, open content, and open knowledge. MIT Press.

Logoze, C., Van de Sompel, H., Nelson, M., \& Warner, S. (2002). The open archives initiative protocol for metadata harvesting. http://www.openarchives.org/OAl/openarchivesprotocol.html

McGreal, R., Anderson, T., Babin, G., Downes, S., Friesen, N., Harrigan, K., et al. (2004). EduSource: Canada's learning object repository network. The International Journal of Instructional Technology and Distance Learning, http://www.itdl.org/Journal/Mar_04/article01.htm.

OECD (2001). Learning to change: ICT in schools (Schooling for Tomorrow Series). OECD.

OECD (2007). Giving knowledge for free: The emergence of Open Educational Resources. OECD.

Yoshii, A., Yamada, T., \& Shimizu, Y. (2008.12). Development of federated search system for sharing learning objects between NIME-glad and overseas gateways. Educational Technology Research, 31 (1-2), 125-132. 